\title{
Commentary on "Can Elimination of Epinephrine in Rhinoplasty Reduce the Side Effects: Introduction of a New Technique"
}

\author{
Jose Abel de la Pena
}

Received: 24 February 2011/ Accepted: 24 February 2011/Published online: 1 April 2011

(c) Springer Science+Business Media, LLC and International Society of Aesthetic Plastic Surgery 2011

There are many steps in the procedures that we perform on a regular basis that are done by protocol. Many of these have been taught from generation to generation of plastic surgeons and have not been studied and revised to determine if they are optimal for obtaining the desired effect or result. The use of epinephrine with local anesthetics in rhinoplasty provides vasoconstriction and prolongs the anesthetic effect, both desirable in this type of surgery.

In this article [1] the authors prove that it is possible to perform a rhinoplasty without the use of epinephrine in a safe and efficient manner and also that it is feasible to perform a rhinoplasty in patients with a contraindication for using epinephrine. What it fails to prove is that the omission of this medication provides a tangible surgical advantage in performing the procedure or that it is detrimental to the patient.

Theoretically, the use of epinephrine provides a timeproven advantage, but as demonstrated in this article, it is not an indispensable requirement to obtain the conditions needed for a good outcome in experienced hands.
Statistically, only operating time was improved and there was no meaningful difference for all other parameters of this study. So recommending ceasing the use of epinephrine cannot be made.

We have a responsibility to our patients to perform all procedures in the safest and most efficient way possible. That is why it is imperative that these types of studies are encouraged, so we are able to omit or apply new or timetested surgical steps.

Disclosure The author declares that he has no conflicts of interest to disclose.

\section{Reference}

1. Kalantar-Hormozi A, Fadaee-Naeeni A, Solaimanpour S, Mozaffari N, Yazdanshenas H, Bazargan-Hejazi S (2011) Can elimination of epinephrine in rhinoplasty reduce the side effects: introduction of a new technique. Aesthetic Plast Surg 35. doi: 10.1007/s00266-011-9673-4
J. A. de la Pena $(\bowtie)$

Institute for Plastic Surgery Mexico City, Hospital Angeles de Interlomas, Vial. de la Barranca s/n-490, Col. Valle de las

Palmas, 52763 Huixquilucan, Edo. Mex, Mexico

e-mail: abeldelapena@plasticsurgery.com.mx 